\title{
Semen Biochemical Components in Varicocele, Leukocytospermia, and Idiopathic Infertility
}

\author{
Giulia Collodel ${ }^{1}$ (D) Cinzia Signorini $^{1} \cdot$ Fabiola Nerucci $^{2} \cdot$ Laura Gambera $^{3} \cdot$ Francesca lacoponi $^{4} \cdot$ Elena Moretti $^{1}$
}

Received: 22 April 2020 / Accepted: 3 July 2020 / Published online: 22 July 2020

(C) The Author(s) 2020

\begin{abstract}
The evaluation of the seminal plasma plays a relevant role in the definition of male infertility and in assisted reproduction outcomes; for this reason, it would be recommended to find biochemical markers able to characterize sperm pathology. In this study, 53 infertile patients (grouped by the presence leukocytospermia, idiopathic infertility, or varicocele) and 10 fertile men were selected. Spermiogram was performed by light microscopy, and sperm ultrastructure was evaluated by transmission electron microscopy (TEM) mathematically elaborated. Testosterone (TESTO), estradiol (E2), ferritin (FERR), iron (Fe), transferrin (TRSF), triglycerides (TRG), cholesterol (CHOL), and isoprostanes ( $\mathrm{F}_{2}$-IsoPs) were detected in seminal plasma. Sperm characteristics and biochemical components were correlated by Spearman's rank correlation coefficient in the whole population and in each group. The levels of TESTO and E2 were positively correlated with sperm quality in particular, and E2 was correlated with fertility index expressing the number of sperm free of ultrastructural defects evaluated by TEM. On the contrary, the indices of iron metabolism (FERR, Fe, and TRSF) were positively associated with low sperm quality and sperm necrosis, particularly in leukocytospermia and varicocele groups, pathologies in which an inflammatory status and oxidative stress condition are present. The study of the seminal plasma composition deserves attention because the levels of the various components seem to be associated with specific reproductive pathologies.
\end{abstract}

Keywords Iron metabolism $\cdot$ Isoprostanes $\cdot$ Leukocytospermia $\cdot$ TEM $\cdot$ Testosterone $\cdot$ Varicocele

\section{Introduction}

Seminal plasma is the protein-rich fluid part of the semen and it plays an important role in sperm metabolism as well as sperm function. The analyses of seminal enzyme activities and the concentrations of biochemical elements have been performed in men and animals [1-4] in order to establish the functions of the different components, because some of them are still obscure.

Giulia Collodel

giulia.collodel@unisi.it

1 Department of Molecular and Developmental Medicine, University of Siena, Policlinico Le Scotte, Viale Bracci 14, 53100 Siena, Italy

2 Division of Clinical Pathology, University Teaching, Hospital of Siena, Siena, Italy

3 Fertility Center, AGI Medica, Siena, Italy

4 Department of Food Safety, Nutrition and Veterinary Public Health, National Institute of Health, Rome, Italy
The evaluation of seminal components may be used to characterized sperm quality and pathological conditions related to male infertility [5]. Many molecules are involved in the pathophysiology of spermatozoa. Iron is an essential trace nutrient that plays an important role in general health and fertility; however, it is highly toxic if accumulated in large quantities. The excess or the deficiency may lead to defective spermatogenesis and to fertility impairment [6]. About this topic, Hashemi et al. [7] reported a negative impact of iron on human sperm motility.

Transferrin is reported to be an iron-binding protein that regulates free iron levels in biological fluid in the body [8]. In the past, Barthelemy et al. [9] proposed that the seminal fluid transferrin, that correlated with sperm concentration, could be considered as a direct index of the functional state of Sertoli cells and seminiferous tubules. Recently, it has been found that transferrin could markedly extend sperm longevity in vitro [10].

Ferritin is an intracellular protein that stores iron in the atoxic form, and it is found in the extracellular environment as a result of cellular synthesis and secretion. Seminal ferritin 
is found in abundance in the seminal plasma, and Sertoli cells produce approximately $70 \%$ of its contents [11].

The investigation of other different semen biochemical components may explain their role in seminal pathologies. Recently, Murgia et al. [12] indicated that seminal fluid metabolome may represent a marker for oligozoospermia. Moreover, the identification of the lipids most closely related to the reproductive success may be of great interest to the scientific community. Cholesterol is a component of the mammalian plasma membrane and its redistribution and depletion from the sperm membrane are the key parts of the spermatozoon's preparation for fertilization [13].

Many hormones are also found among the constituents of seminal plasma. When compared with blood serum levels, concentrations of testosterone in seminal plasma are lower by almost one order of magnitude, while estradi$\mathrm{ol}$ is in some instances higher [14]. Other authors [15, 16] observed that the seminal balance between estradiol and testosterone provides a valuable biological indicator for predicting the spermatogenic state.

Our previous paper reported that increased albumin, total proteins, ferritin levels, and $\gamma$-glutamyl transferase and creatine kinase activities concomitant with decreased amounts of alkaline phosphatase and folic acid were associated with reduced sperm parameters. These results suggested their possible involvement in male infertility [17]. In humans, many pathological conditions as varicocele, urogenital infections, leukocytospermia, and inflammation influence sperm quality as well as seminal compositions. Oxidative stress in seminal fluid may be the common denominator responsible of male infertility associated with these diseases; the increased presence of reactive oxygen species (ROS) affects seminal components and it causes alterations in sperm lipids, proteins, and DNA with consequent sperm impairment [18].

Changes in the concentrations of the trace elements in human seminal plasma may be related to sperm quality since they are involved in the maintenance of the pro-/antioxidative balance in ejaculate [19]. In the presence of oxidative stress, a non-enzymatic oxidative damage in sperm plasma membranes, particularly rich in polyunsaturated fatty acid, may generate prostaglandin-like end-products known as isoprostanes (IsoPs). Seminal $\mathrm{F}_{2}$-isoprostanes ( $\mathrm{F}_{2}$-IsoPs), a specific class of IsoPs, have been indicated as a marker of sperm immaturity in semen of infertile patients with varicocele [20].

To clarify the role of specific biochemical semen components in sperm quality, we have investigated the levels of cholesterol (CHOL), estradiol (E2), testosterone (TESTO), iron $(\mathrm{Fe})$, ferritin (FERR), transferrin (TRSF), and triglycerides (TRG) in seminal fluid of fertile and infertile men grouped according to their reproductive pathologies. Isoprostane $\left(\mathrm{F}_{2}\right.$-IsoP $)$ amount was also dosed. Semen analysis was performed by light and transmission electron microscopy
(TEM) mathematically elaborated. As a result, all the variables were correlated and compared.

\section{Materials and Methods}

\section{Patients}

In this research, from January 2018 through December 2018, we selected 53 Italian infertile patients (aged 28-39) attending AGI Medica, Fertility Center lab for semen analysis. Infertile patients did not obtain pregnancy after 2 years of unprotected sexual intercourses; the female factor was not present.

The infertile patients were grouped as follows: 16 idiopathic infertile patients, 19 patients with varicocele, and 18 patients with abacterial leukocytospermia. Leukocytospermia has been defined as reported in WHO guidelines [21]. The varicocele group was composed by patients who underwent both physical examination and scrotal color Doppler ultrasonography analysis carried out in laboratory other than ours. Patients with subclinical and grade 1 varicocele were not included in the study.

Inclusion selection criteria were non-azoospermic men, normal karyotype, BMI $<25 \mathrm{~kg} / \mathrm{m}^{2}$, normal serum hormonal levels (follicle-stimulating hormone, luteinizing hormone, testosterone, prolactin), and absence of genitourinary infections. We did not include carriers of chromosome Y microdeletions and patients showing germ cells in their ejaculates. Patients with chronic diseases and receiving radiotherapy, chemotherapy, and medication were excluded. Selected men did not take an oral antioxidant supplement for at least 4 months before the study. Men with a history of recreational drug use and alcohol consumption were not selected.

Only individuals with moderate and heavy smoking habit ( $>10$ cigarettes/day) were excluded [22].

The fertile group was composed of 10 individuals (aged 26-40) which fathered at least one child in the last 3 years. The fertile subjects were not affected by infections and/or anatomical problems.

All selected men provided an informed written consent before the inclusion on this research where the aims of the study were accurately described.

\section{Semen Analysis}

Semen analysis was performed according to WHO guidelines [21]. Briefly, specimens were collected by masturbation after 3-5 days of sexual abstinence and analyzed after liquefaction for $30 \mathrm{~min}$ at $37{ }^{\circ} \mathrm{C}$. Conventional semen parameters were determined: volume, $\mathrm{pH}$, sperm concentration, motility, and vitality. The sperm morphology was assessed by the stain-coated testsimplets slides (Origio, Italy). Peroxidase stain was used to identify the 
leukocytes in semen samples. A leukocyte concentration $\geq 1$ million cell $/ \mathrm{ml}$ was considered abnormal [21].

After semen evaluation, all the samples were divided into three aliquots to be used for different analyses.

An aliquot was processed for transmission electron microscopy, and the seminal volume was doubled with cold Karnovsky fixative and maintained at $4{ }^{\circ} \mathrm{C}$ for $2 \mathrm{~h}$. Then samples were centrifuged at $200 \mathrm{~g}$ for $15 \mathrm{~min}$, the supernatant was discarded, and sperm cells were washed in $0.1 \mathrm{~mol} / 1$ cacodylate buffer ( $\mathrm{pH} 7.2$ ) for $12 \mathrm{~h}$, postfixed in $1 \%$ buffered osmium tetroxide for $1 \mathrm{~h}$ at $4{ }^{\circ} \mathrm{C}$, and it was washed again in $0.1 \mathrm{~mol} / \mathrm{l}$ cacodylate buffer; the samples were dehydrated in a graded ethanol series and embedded in Epon Araldite. Ultrathin sections were cut with a Supernova ultramicrotome (Reickert Jung, Vienna, Austria), mounted on copper grids, stained with uranyl acetate and lead citrate, and at least observed and photographed with a Philips CM12 (Philips Scientifics, Eindhoven, The Netherlands) at the CE.M.E, CNR (Via Madonna del Piano, 10, 50019 Sesto Fiorentino, Italy). Three hundred sperm sections were analyzed from each sample. The TEM data was elaborated using a mathematical formula [23] which provides numerical scores such as fertility index (number of sperm free of structural defects in a semen sample) and percentage of sperm pathologies such as immaturity, apoptosis, and necrosis [24], defined by distinctive ultrastructural characteristics. Sperm immaturity include the presence of cytoplasmic droplets, altered acrosomes, roundish or elliptical nuclei with uncondensed chromatin. Marginated chromatin, translucent vacuoles included in cytoplasmic residues, swollen and badly assembled mitochondria are ultrastructural indicators of apoptosis. Sperm with reacted or absent acrosome, misshaped nuclei with disrupted chromatin, broken plasma membrane, and poor axonemal and periaxonemal cytoskeletal structures are affected by necrosis.

\section{Clinical Biochemistry Determinations}

A semen aliquot of each ejaculate was centrifuged at $12,000 \mathrm{~g}$ to obtain a sample free of cells as reported by Feng et al. [1], and the seminal plasma was collected by micropipette and stored in 2-ml cryotubes at $-80{ }^{\circ} \mathrm{C}$ until use, then thawed at room temperature. Seminal plasma samples $(1 \mathrm{ml}$ at room temperature) were tested using a COBAS 8000 modular analyzer (Roche Diagnostics, Mannheim, GmbH, Germany) by means of two analytical modules: C702, the high-throughput clinical chemistry module, and E602, the immunoassay module.

We measured the following parameters in seminal plasma: iron $(\mathrm{Fe}, \mu \mathrm{g} / \mathrm{dl})$, triglycerides (TRG, $\mathrm{mg} / \mathrm{dl})$, cholesterol (CHOL, mg/dl), transferrin (TRSF, mg/dl), estradiol (E2, pg/ $\mu \mathrm{l}$ ), ferritin (FERR, ng/ml), testosterone (TESTO, ng/ml). For the analytes measured in module C702 (Fe, TRG, CHOL, TRSF), COBAS 8000 calibration was done with the human lyophilized serum Calibrator C.f.a.s. (Roche Diagnostics, Mannheim, GmbH, Germany). The C.f.a.s. (calibrator for automated systems) is a universal calibrator for adjusting most photometric methods.

Human lyophilized serum PreciControl ClinChem level 1 was used as normal control and PreciControl ClinChem level 2 was used as pathologic control (Roche Diagnostics, Mannheim, GmbH, Germany).

For the analytes measured in module E602 (FERR, E2, TESTO), we used specific calibrator for each analyte (Roche Diagnostics, Mannheim, GmbH Germany). PreciControl Varia levels 1 and 2 (Roche 470 Diagnostics, Mannheim, GmbH, Germany), PreciControl Universal levels 1 and 2 (Roche Diagnostics, Mannheim, GmbH, Germany), and PreciControl Tumor Marker levels 1 and 2 (Roche 470 Diagnostics, Mannheim, GmbH, Germany) were used as normal and pathologic controls respectively.

\section{$F_{2}$-Isoprostane Determination}

An aliquot of each semen sample was centrifuged at $200 \mathrm{~g}$ for $15 \mathrm{~min}$, then the pellet was discarded. Seminal sample, devoid of cells, was supplemented with butylated hydroxytoluene (BHT, final concentration $100 \mu \mathrm{M}$ ) and was stored at $-80^{\circ} \mathrm{C}$ until use.

$\mathrm{F} 2$-isoprostanes $\left(\mathrm{F}_{2}\right.$-IsoPs $)$ are originally formed in situ, when the fatty acid precursor is esterified in phospholipids and subsequently released in biological fluids in a freeform through a process likely mediated by a phospholipase $\mathrm{A}_{2}\left(\mathrm{PLA}_{2}\right)$ activity(ies) [25]. Here, total (free plus esterified) $\mathrm{F}_{2}$-IsoPs were analyzed by gas chromatography/negative-ion chemical ionization-tandem mass spectrometry (GC/NICI-MS/MS). After collection, as previously reported, BHT was added in order to avoid unspecific oxidation processes. For each sample, basic hydrolysis was carried out by incubation at $45{ }^{\circ} \mathrm{C}$ for $45 \mathrm{~min}$ in the presence of $1 \mathrm{~N} \mathrm{KOH}$; at the end of the incubation, $\mathrm{HCl} 1 \mathrm{~N}$ was added to stabilize a value of $\mathrm{pH}$ 3.0. At this stage, in each sample, tetradeuterated derivative of prostaglandin $\mathrm{F}_{2 \alpha}\left(\mathrm{PGF}_{2 \alpha}\right.$-d4) $(500 \mathrm{pg})$ was added as an internal standard, and solid-phase extractions were consecutively carried out on an octadecylsilane (C18) cartridge and an aminopropyl $\left(\mathrm{NH}_{2}\right)$ cartridge. The $\mathrm{NH}_{2}$ final eluate was derivatized to convert the carboxyl group of $\mathrm{F}_{2}$-IsoPs into pentafluorobenzyl ester and the hydroxyl group into trimethylsilyl ethers [26]. For 8 -iso- $\mathrm{PGF}_{2 \alpha}$, also referred as $15-\mathrm{F}_{2} \mathrm{t}-\mathrm{IsoP}$, one of the most represented isomers of $\mathrm{F}_{2}$-IsoPs, the product ion at $\mathrm{m} / \mathrm{z} 299$, derived from the $[\mathrm{M}-181]^{-}$precursor ion $(\mathrm{m} / \mathrm{z} 569)$, was detected and referred to the internal standard $\mathrm{PGF}_{2 \alpha}-\mathrm{d} 4(\mathrm{~m} / \mathrm{z}, 573 \rightarrow \mathrm{m} / \mathrm{z}$ 303) [27]. 


\section{Statistical Analysis}

Data were reported as medians and interquartile range (IQR $75^{\circ}-25^{\circ}$ centile). The comparisons between groups (fertile, idiopathic infertility, varicocele, leukocytospermia) were evaluated by Kruskal-Wallis test followed, only for significant cases, by Dunn's post hoc test for multiple comparisons. Spearman rho $(R)$ coefficient was calculated to measure the correlation between variables for all enrolled patients and separately for each group. A $P$ value $<0.05$ (two-tailed) was considered statistically significant. All analyses were performed by IBM SPSS Statistics Software v.25.

\section{Results}

Semen characteristics of the infertile patients with leukocytospermia, idiopathic infertility, varicocele, and fertile group are reported in Table 1. Idiopathic infertility group significantly differed in almost all evaluated sperm characteristics from fertile men. Patients with leukocytospermia showed a significant decrease in sperm motility, normal sperm morphology, and vitality with respect to fertile patients, and higher percentage of necrosis (Fig. 1a) compared to that observed in fertile and varicocele groups $(P<.001)$. Finally, in varicocele patients, immaturity percentage was significantly elevated $(P<.001)$ with respect to that detected in fertile, idiopathic infertility, and leukocytospermia groups (Table 1).

The medians and interquartile ranges of seminal levels of $\mathrm{F}_{2}$-IsoPs, CHOL, E2, TESTO, Fe, FERR, TRSF, and TRIG are shown in Table 2.
$\mathrm{F}_{2}$-IsoP level, a reliable indicator of oxidative stress, was significantly increased in varicocele group compared to leukocytospermia, idiopathic infertility, and fertile groups $(P<.001)$. The values of CHOL, E2, TESTO, TRSF, and TRG did not show differences in the considered groups. In leukocytospermia group, the concentration of FERR was significantly increased than that observed in fertile, varicocele, and idiopathic infertility groups $(P<.001)$ and the Fe level was higher than that detected in idiopathic infertility group $(P<.05)$.

In order to understand the possible correlations among the studied variables, we used the Spearman rank correlation coefficient considering the whole population of interest (Table 3). Regarding biochemical components, $\mathrm{F}_{2}$-IsoP levels correlated positively with sperm immaturity (Fig. 1b; $P<.01)$. Positive correlations between the TRSF $(P<.01)$ and TRG $(P<.05)$ levels and sperm concentration were observed. FERR showed negative correlations with sperm morphology, vitality, and fertility index $(P<.05)$ and positive correlations with sperm necrosis $(P<.01)$ and $\mathrm{Fe}(P<.05)$; Fe compound also positively correlated with sperm necrosis $(P<.05)$. Interestingly, TESTO displayed positive correlations with the sperm vitality $(P<.05)$ and E2 level $(P<.01)$ and negative correlations with TRG $(P<.01)$ and sperm apoptosis (Fig. 1c; $P<.05$ ).

Different correlations between biochemical indices and sperm parameters were analyzed in each studied group (Table 4, Spearman's rank correlation coefficient).

In fertile men, E2 positively correlated with fertility index $(P<.05)$ and TESTO $(P<.01)$; CHOL positively correlated with TRG $(P<.001)$.
Table 1 Medians and interquartile ranges (IQR $75^{\circ}-25^{\circ}$ centile) of semen parameters evaluated by light microscopy, sperm apoptosis, necrosis, immaturity, and fertility index evaluated by transmission electron microscopy in semen samples of 63 men divided into 4 groups (leukocytospermia, L; idiopathic infertility, I; varicocele, V; fertile men, F) according to their clinical condition. Statistics are also reported: $* P<.05, * * P<.01, * * * P<.001$

\begin{tabular}{|c|c|c|c|c|c|c|}
\hline \multirow[t]{2}{*}{ Variables } & \multicolumn{4}{|l|}{ Diagnosis } & \multicolumn{2}{|l|}{ Statistics } \\
\hline & $\begin{array}{l}\text { Leukocytospermia } \\
\text { (L no. 18) }\end{array}$ & $\begin{array}{l}\text { Idiopathic infertility } \\
\text { (I no. 16) }\end{array}$ & $\begin{array}{l}\text { Varicocele } \\
\text { (V no. 19) }\end{array}$ & $\begin{array}{l}\text { Fertile men } \\
(\mathrm{F} \text { no. } 10)\end{array}$ & $\begin{array}{l}\text { Kruskal-Wallis } \\
(P \text { value })\end{array}$ & $\begin{array}{l}\text { Multiple } \\
\text { comparisons }\end{array}$ \\
\hline Volume (ml) & $3.25(2.13)$ & $3.75(1.50)$ & $4.00(0.50)$ & $4.25(1.13)$ & 0.068 & \\
\hline Sperm $\mathrm{ml} \times 10^{6}$ & $40.50(68.35)$ & $6.50(28.56)$ & $38.50(36.38)$ & $118.50(128.06)$ & $* * *$ & F vs I \\
\hline Progressive motility $\%$ & $25.50(15.50)$ & $26.50(19.75)$ & $25.00(24.00)$ & $48.00(30.25)$ & $*$ & F vs I; F vs L \\
\hline normal morphology $\%$ & $9.00(3.25)$ & $7.50(4.75)$ & $10.00(7.00)$ & $16.00(7.75)$ & $* * *$ & F vs I; F vs L \\
\hline Vitality $\%$ & $65.00(30.50)$ & $66.50(28.75)$ & $75.00(21.00)$ & $85.00(12.75)$ & $* *$ & F vs I; F vs L \\
\hline Apoptosis \% & $6.48(3.42)$ & $9.11(6.83)$ & $4.13(1.65)$ & $4.13(0.86)$ & $* * *$ & F vs I; I vs V \\
\hline Necrosis \% & $44.00(19.95)$ & $37.74(10.72)$ & $34.24(19.65)$ & $30.05(11.11)$ & $* * *$ & F vs L; L vs V \\
\hline Immaturity \% & $50.65(23.04)$ & $53.89(13.15)$ & $75.09(10.89)$ & $47.20(8.26)$ & $* * *$ & F vs V; I vs V; L vs V \\
\hline Fertility index & $266,546(785,647)$ & $6811(420,986)$ & $287,471(1,497,605)$ & $4,025,583(5,444,460)$ & $* * *$ & F vs I; F vs L; F vs V \\
\hline
\end{tabular}





Fig. 1 TEM micrographs of longitudinal sections of spermatozoa. In a, two necrotic spermatozoa are shown, the chromatin $(\mathrm{dCh})$ appears disrupted, the plasma membranes are broken (arrow), and the axonemal components are absent $(\mathrm{aAx})$. An immature sperm is represented in $\mathbf{b}$, the chromatin is uncondensed ( $\mathrm{uCh})$, the acrosome (A) reduced and far from

the nucleus, the tail is coiled into a cytoplasmic residue (CR), and mitochondria (M) are disassembled. An apoptotic sperm is shown in $\mathbf{c}$, the chromatin is marginated ( $\mathrm{mCh}$ ), the tail is coiled but the axonemal components are well assembled (Ax); acrosome (A); and cytoplasmic residue (CR). a-c Bar, $1 \mu \mathrm{m}$

In idiopathic infertility group, we did not observe significant correlations between seminal plasma components and sperm characteristics.

In leukocytospermia group, E2 positively correlated with TESTO $(P<.01)$ and TESTO negatively correlated with sperm necrosis $(P<.05)$; both TESTO and E2 negatively correlated with FERR $(P<.05)$. TRSF had positive correlations with CHOL and TRG (both $P<.01$ ). Sperm immaturity positively correlated with $\mathrm{F}_{2}$-IsoPs $(P<.05)$. Sperm necrosis is positively correlated with CHOL $(P<.05)$.

In infertile patients affected by varicocele, E2 showed positive correlation with TESTO $(P<.01)$, and both E2 and
TESTO negatively correlated with TRSF (respectively $P<.01 ; P<.05)$. FERR displayed a positive correlation with sperm necrosis $(P<.05)$ and negative correlation with sperm motility $(P<.05)$. Finally, $\mathrm{F}_{2}$-IsoPs had a positive relationship with sperm immaturity $(P<.01)$.

\section{Discussion}

The evaluation of the seminal plasma composition may play a relevant role in the definition of male infertility and in assisted reproduction outcomes [28]; for this reason, it would be
Table 2 Medians and interquartile ranges (IQR $75^{\circ}-25^{\circ}$ centile) of isoprostanes ( $\mathrm{F}_{2}$-IsoPs), cholesterol (CHOL), estradiol (E2), testosterone (TESTO), iron (Fe), ferritin (FERR), transferrin (TRSF), and triglycerides (TRG) assayed in semen samples of 63 men divided into 3 groups according to their clinical condition (leukocytospermia, L; idiopathic infertility, I; varicocele, V; fertile men, F). Statistics are also reported: $* P<.05, * * * P<.001$

\begin{tabular}{|c|c|c|c|c|c|c|}
\hline \multirow[t]{2}{*}{ Variables } & \multicolumn{4}{|l|}{ Diagnosis } & \multicolumn{2}{|l|}{ Statistics } \\
\hline & Leukocytospermia (L no. 18) & $\begin{array}{l}\text { Idiopathic infertility } \\
\text { (I no. 16) }\end{array}$ & $\begin{array}{l}\text { Varicocele } \\
\text { (V no. 19) }\end{array}$ & $\begin{array}{l}\text { Fertile men } \\
(\mathrm{F} \text { no. } 10)\end{array}$ & $\begin{array}{l}\text { Kruskal-Wallis } \\
(P \text { value })\end{array}$ & Multi-comparison test \\
\hline $\mathrm{F}_{2}$-IsoPs $(\mathrm{ng} / \mathrm{ml})$ & $27.22(31.50)$ & $7.03(32.63)$ & $76.16(55.46)$ & $8.56(17.42)$ & $* * *$ & I vs V; F vs V; L vs V \\
\hline CHOL (mg/dl) & $22.00(14.75)$ & $19.00(10.00)$ & $17.00(9.00)$ & $19.00(13.75)$ & 0.326 & \\
\hline $\mathrm{E} 2(\mathrm{pg} / \mu \mathrm{l})$ & $45.83(27.77)$ & $55.63(16.57)$ & $58.93(29.24)$ & $47.09(19.36)$ & 0.154 & \\
\hline TESTO (ng/ml) & $0.94(0.37)$ & $1.04(1.33)$ & $1.10(2.03)$ & $1.26(1.57)$ & 0.264 & \\
\hline $\mathrm{Fe}(\mu \mathrm{g} / \mathrm{dl})$ & $23.00(16.50)$ & $13.00(8.25)$ & $15.00(10.00)$ & $14.50(11.00)$ & * & I vs L \\
\hline FERR (ng/ml) & $337.2(179.4)$ & $233.9(91.3)$ & $238.2(77.3)$ & $158.4(75.8)$ & $* * *$ & L vs F; L vs V; L vs I \\
\hline TRSF (mg/dl) & $1.50(7.00)$ & $0.50(1.00)$ & $3.64(3.00)$ & $0.50(3.00)$ & 0.117 & \\
\hline TRG (mg/dl) & $99.50(181.75)$ & $7.00(59.25)$ & $2.00(126.00)$ & $56.50(164.50)$ & 0.053 & \\
\hline
\end{tabular}




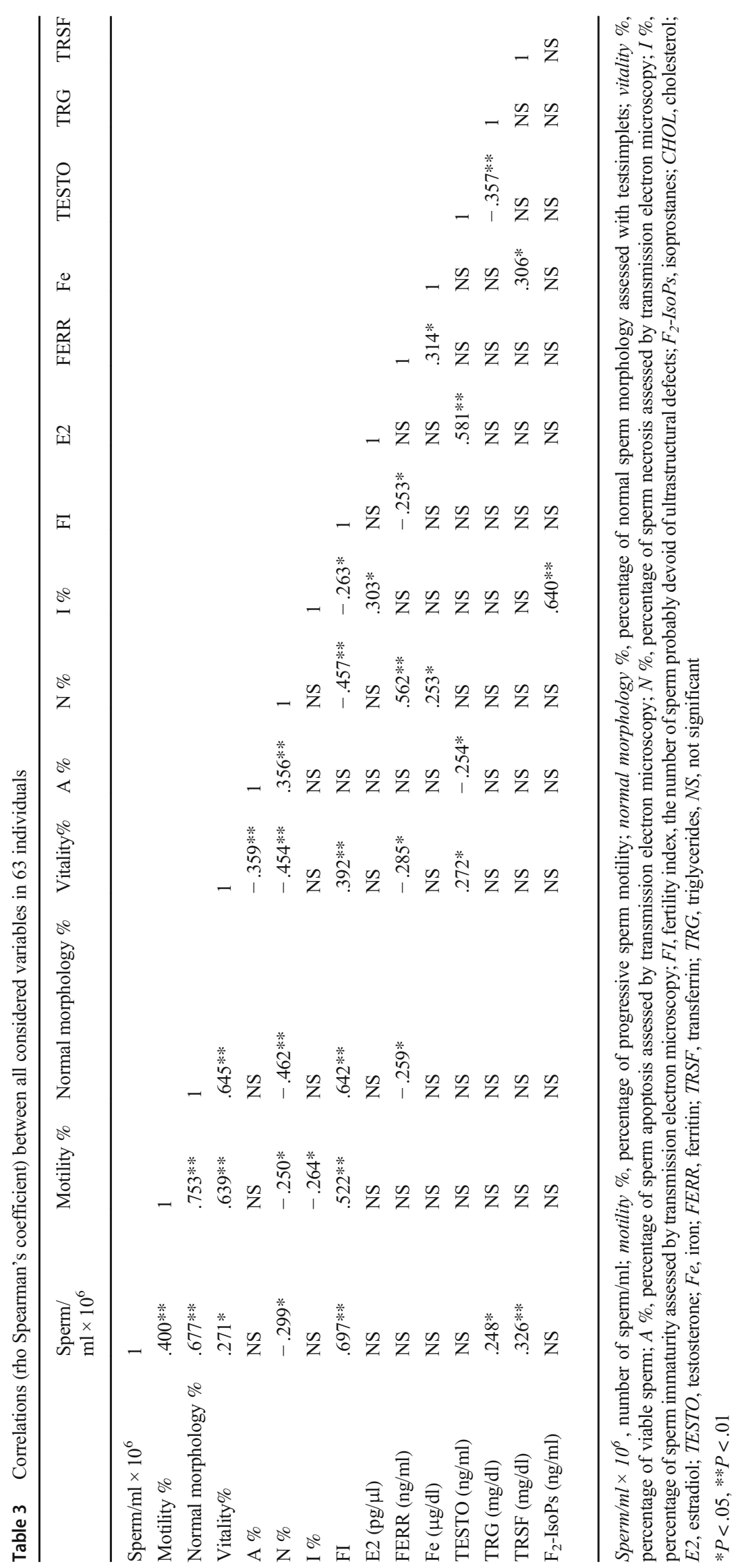




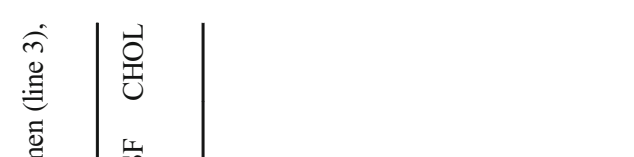

. 合

产

完




recommended to find biochemical markers able to characterize sperm pathology [29]. Our and other groups have stressed on the importance of the research of seminal indicators in the different male infertility conditions [30-33]. In our recent paper, we suggested that some seminal biochemical components may be associated with pathological conditions of human semen. Patients with sperm vitality $\leq 5$ th percentile showed increased albumin concentration and creatine kinase activity. The presence of germ cells in semen was associated with high values of FERR; the alkaline phosphatase activity and folic acid were decreased in hyperviscous semen samples [17]. There are several studies focused on the proteomic composition of human seminal plasma, which may be linked to specific pathways of infertility [3, 34]. Mirnamniha et al. [35] reported that the deficiency of trace elements such as calcium copper, manganese, magnesium, zinc, and selenium is associated with impaired spermatogenesis, altered levels of sex hormones, seminal oxidative stress, inflammation, and apoptosis. Recently, Murgia et al. [12] detected altered levels of fructose, myo-inositol, aspartate, and choline in oligozoospermic men.

In this paper, the data regarding the analyzed variables suggested some intriguing hypotheses. The level of TESTO did not differ in the examined groups but it was strongly positively correlated with sperm quality and with E2; in turn, E2 level positively correlated with fertility index expressing the number of sperm free of ultrastructural defects evaluated by TEM. These results confirmed that these hormones have a relevant positive influence in sperm physiology and the seminal balance between estrogenic and androgenic actions is essential for maintaining spermatogenesis and male fertility $[15,16,36]$. In agreement with our data, Thanaboonyawat et al. [37] demonstrated that in vitro supplementation of TESTO determined a significant retardation in the normal reduction of sperm motility over time. Moreover, the effects of E2 on human sperm have been associated with an increased motility, oocyte penetration, longevity, oxygen intake, and metabolization of exogenous substrates [38-40]. The correlation between these hormones is justified by the fact that in adult human and animal testis, TESTO is converted to E2 by aromatase (CYP19A1), the ubiquitous NADPH cytochrome P450 reductase enzyme principally found in spermatids and mature sperm in seminiferous tubules and Leydig cells [41]. A further confirmation that TESTO is a positive marker of semen quality is suggested by its negative correlation with sperm necrosis.

On the contrary, the indices of iron metabolism (FERR, Fe, and TRSF) were positively associated with low sperm quality and negatively with TESTO and E2 levels, particularly in leukocytospermia and varicocele groups, pathologies in which an inflammatory status and oxidative stress condition are generally present [42]. 
The relationship among TESTO, FERR, and inflammation is well known in the literature [43-46] even if the determinations are performed in blood serum. In our study, they were detected in human seminal plasma and these associations were confirmed suggesting the involvement of cytokines [43] and ROS [47] also in the seminal fluid from patients with varicocele or leukocytospermia. It is known that an increased number of leukocytes are positively associated with altered sperm characteristics, sperm necrosis, intracellular ROS [30, 48, 49], and level of $\mathrm{Fe}$ in the seminal plasma of men with different fertility potentials [50]. Overload of $\mathrm{Fe}$ may cause disturbances to spermatogenesis as well as to crucial sperm cell structures accompanied by oxidative stress and cell death $[6,50]$.

FERR has been localized in human Sertoli and Leydig cells $[51,52]$ and an increased level of FERR was detected in human semen concomitantly to the presence of germ cells [17]. Seminal fluid TRSF values were proposed as a reliable clinical index for determining the functional status of Sertoli cells or seminiferous tubules [9]. In mice, TRSF overexpression had negative effects on testicular function and TRSF level required strict regulation in the testis [53]. In our paper, the negative correlation between TRSF and TESTO and E2 in varicocele patients may suggest a role of TRSF as an index of testis malfunctioning since it is well known that varicocele can alter Sertoli cell function and consequently influence the TSRF level.

This study confirmed the close relationship between increased levels of $\mathrm{F}_{2}$-IsoP varicocele and sperm immaturity observed also in previous studies [20,54]; since these studies included different patient populations, the semen level of $\mathrm{F}_{2^{-}}$ IsoPs can be considered a semen biochemical marker of sperm immaturity and varicocele.

Noteworthy is the behavior of CHOL and TRG that in the first analysis seems to be irrelevant, either as marker of pathologies either in correlations performed in the whole studied population. When the correlations between variables were carried out in each group separately, we observed that in the case of leukocytospermia, CHOL and TRG positively correlated with sperm necrosis. Previous studies suggested that elevated lipid levels in seminal plasma might have adverse effect on sperm quality [55], and seminal plasma TRG and total CHOL levels had a positive correlation with sperm DNA fragmentation [56]. Also increased lipid serum levels play a negative influence on sperm quality [57].

Patients with idiopathic infertility showed an altered sperm quality as the other infertile groups; however, they did not show correlations between semen parameters and seminal plasma markers highlighted for leukocytospermia and varicocele groups. This observation could be explained by the intrinsic meaning of idiopathic infertility since it is difficult to group patients with homogeneous characteristics. Indeed, in semen of many men who are currently diagnosed as having unexplained male infertility, oxidative stress was detected, but other genetic, epigenetic, or environmental unknown causes could be supposed $[58,59]$.

\section{Conclusions}

In conclusion, infertile men with varicocele, leukocytospermia, and idiopathic infertility differed in seminal fluid composition. TESTO and E2 levels appeared to be necessary for a regular spermatogenesis and play a role in sperm quality; FERR, Fe, and TRSF appeared to be linked to inflammatory conditions and sperm necrosis as well as $\mathrm{F}_{2}$-IsoPs to sperm immaturity. We are aware that the number of cases is low and should be extended in a future study; however, these preliminary data indicated that the seminal plasma composition deserves attention because the levels of the various components seem to be associated with specific reproductive pathologies.

The knowledge of the role of seminal molecules in male infertility will help in the development of targeted treatments.

Acknowledgments Open access funding provided by Università degli Studi di Siena within the CRUI-CARE Agreement. We sincerely acknowledged the assistance given by Dr. Maria Cristina Salvatici, CE.M.E, CNR (Via Madonna del Piano, 10, 50019 Sesto Fiorentino, Italy), in this study.

Authors' Contributions All the authors approved the submitted version of this paper and gave substantial contributions to the research design and drafting or critically revising the paper. In particular: Giulia Collodelpaper writing, research design, TEM analysis, data interpretation; Cinzia Signorini-isoprostane determination, data interpretation; Fabiola Nerucci-biochemical component determination, data interpretation; Francesca Iacoponi-statistical analysis; Laura Gambera-patient recruitment, semen analysis, data interpretation; Elena Moretti-patient selection, TEM analysis, data interpretation, research design.

Funding Information This research was supported by the Plan Support to the Research (PSR) 2019, Department of Molecular and Developmental Medicine, University of Siena, Italy.

Data Availability The datasets used and/or analyzed during the current study are available from the corresponding author on reasonable request.

\section{Compliance with Ethical Standards}

Conflict of Interest The authors declare that they have no conflict of interest.

Patient Consent All participants were informed and gave written consent prior to the collection of samples.

Open Access This article is licensed under a Creative Commons Attribution 4.0 International License, which permits use, sharing, adaptation, distribution and reproduction in any medium or format, as long as you give appropriate credit to the original author(s) and the source, provide a link to the Creative Commons licence, and indicate if changes were made. The images or other third party material in this article are included in the article's Creative Commons licence, unless indicated otherwise in a 
credit line to the material. If material is not included in the article's Creative Commons licence and your intended use is not permitted by statutory regulation or exceeds the permitted use, you will need to obtain permission directly from the copyright holder. To view a copy of this licence, visit http://creativecommons.org/licenses/by/4.0/.

\section{References}

1. Feng RX, Lu JC, Zhang HY, Lü NQ. A pilot comparative study of 26 biochemical markers in seminal plasma and serum in infertile men. Biomed Res Int. 2015;2015:805328.

2. Zhang HY, Lu JC, Feng RX. Correlations of 24 biochemical markers in seminal plasma with routine semen parameters. Zhonghua Nan Ke Xue. 2015;21(12):1087-92.

3. Camargo M, Intasqui P, Bertolla RP. Understanding the seminal plasma proteome and its role in male fertility. Basic Clin Androl. 2018;28:6

4. Recuero S, Fernandez-Fuertes B, Bonet S, Barranco I, Yeste M. Potential of seminal plasma to improve the fertility of frozenthawed boar spermatozoa. Theriogenology. 2019;137:36-42.

5. Vickram AS, Kamini AR, Das R, Pathy MR, Parameswari R, Sridharan TB. Validation of artificial neural network models for predicting biochemical markers associated with male infertility. Syst Biol Reprod Med. 2016;62(4):258-65.

6. Tvrda E, Peer R, Sikka SC, Agarwal A. Iron and copper in male reproduction: a double-edged sword. J Assist Reprod Genet. 2015;32(1):3-16.

7. Hashemi MM, Behnampour N, Nejabat M, Tabandeh A, GhaziMoghaddam B, Joshaghani HR. Impact of seminal plasma trace elements on human sperm motility parameters. Rom J Intern Med. 2018;56(1):15-20

8. Kleven MD, Jue S, Enns CA. The transferrin receptors, TfR1 and TfR2 bind transferrin through differing mechanisms. Biochemistry. 2018;57(9):1552-9.

9. Barthelemy C, Khalfoun B, Guillaumin JM, Lecomte P, Bardos P. Seminal fluid transferrin as an index of gonadal function in men. $\mathrm{J}$ Reprod Fertil. 1998;82(1):113-8.

10. Matsuzaki M, Mizushima S, Dohra H, Sasanami T. Expression of transferrin and albumin in the sperm-storage tubules of Japanese quail and their possible involvement in long-term sperm storage. $\mathrm{J}$ Poult Sci. 2020;57(1):88-96.

11. Silva GP, De La Vega Elena CD, Pirani Carneiro F, Russomano Veiga JP. Effect of systemic inflammation on level of ferritin seminal in chronic renal male patient undergoing hemodialysis. Int Arch Med. 2014;7:23.

12. Murgia F, Corda V, Serrenti M, et al. Seminal fluid metabolomic markers of oligozoospermic infertility in humans. Metabolites. 2020;10(2):E64.

13. Leahy T, Gadella BM. New insights into the regulation of cholesterol efflux from the sperm membrane. Asian J Androl. 2015;17(4): 561-7.

14. Vitku J, Kolatorova L, Hampl R. Occurrence and reproductive roles of hormones in seminal plasma. Basic Clin Androl. 2017;27:19.

15. O'Donnell L, Robertson KM, Jones ME, Simpson ER. Estrogen and spermatogenesis. Endocr Rev. 2001;22(3):289-318.

16. Zhang Q, Bai Q, Yuan Y, Liu P, Qiao J. Assessment of seminal estradiol and testosterone levels as predictors of human spermatogenesis. J Androl. 2010;31(2):215-20.

17. Collodel G, Nerucci F, Signorini C, Iacoponi F, Moretti E. Associations between biochemical components of human semen with seminal conditions. Syst Biol Reprod Med. 2019;65(2):15563
18. Benedetti S, Tagliamonte MC, Catalani S, Primiterra M, Canestrari F, De Stefani S, et al. Differences in blood and semen oxidative status in fertile and infertile men, and their relationship with sperm quality. Reprod BioMed Online. 2012;25(3):300-6.

19. Nenkova G, Petrov L, Alexandrova A. Role of trace elements for oxidative status and quality of human sperm. Balkan Med J. 2017;34(4):343-8.

20. Collodel G, Moretti E, Longini M, Pascarelli NA, Signorini C. Increased F2-isoprostane levels in semen and immunolocalization of the 8-Iso prostaglandin F $\alpha$ in spermatozoa from infertile patients with varicocele. Oxidative Med Cell Longev. 2018;2018: 7508014.

21. World Health Organization. WHO laboratory manual for the examination and processing of human semen. 5th ed. Geneva: WHO Press; 2010

22. Collodel G, Capitani S, Iacoponi F, Federico MG, Pascarelli NA, Moretti E. Retrospective assessment of potential negative synergistic effects of varicocele and tobacco use on ultrastructural sperm morphology. Urology. 2009;74(4):794-9.

23. Baccetti B, Bernieri G, Burrini AG, Collodel G, Crisà N, Mirolli M, et al. Notulae Seminologicae. 5. Mathematical evaluation of interdependent submicroscopic sperm alterations. J Androl. 1995;16(4): 356-71.

24. Collodel G, Moretti E. Morphology and meiotic segregation in spermatozoa from men of proven fertility. J Androl. 2008;29(1): $106-14$.

25. Stafforini DM, Sheller JR, Blackwell TS, Sapirstein A, Yull FE, McIntyre TM, et al. Release of free F2-iso-prostanes from esterified phospholipids is catalyzed by intracellular and plasma plateletactivating factor acetylhydrolases. J Biol Chem. 2006;281(8): 4616-23.

26. Signorini C, De Felice C, Durand T, et al. Relevance of 4-F(4t)neuroprostane and 10-F(4t)-neuroprostane to neurological diseases. Free Radic Biol Med. 2018;115:278-87.

27. Signorini C, Cardile V, Pannuzzo G, Graziano ACE, Durand T, Galano JM, et al. Increased isoprostanoid levels in brain from murine model of Krabbe disease - relevance of isoprostanes, dihomoisoprostanes and neuroprostanes to disease severity. Free Radic Biol Med. 2019;139:46-54.

28. Cooper TG, Noonan E, von Eckardstein S, Auger J, Baker HWG, Behre HM, et al. World Health Organization reference values for human semen characteristics. Hum Reprod Update. 2010;16(3): $231-45$.

29. Lazzarino G, Listorti I, Muzii L, Amorini AM, Longo S, di Stasio E, et al. Low-molecular weight compounds in human seminal plasma as potential biomarkers of male infertility. Hum Reprod. 2018;33(10):1817-28.

30. Collodel G, Moretti E, Micheli L, Menchiari A, Moltoni L, Cerretani D. Semen characteristics and malondialdehyde levels in men with different reproductive problems. Andrology. 2015;3: $280-6$.

31. Micheli L, Cerretani D, Collodel G, Menchiari A, Moltoni L, Fiaschi AI, et al. Evaluation of enzymatic and non-enzymatic antioxidants in seminal plasma of men with genitourinary infections, Varicocele and Idiopathic Infertility. Andrology. 2016;4(3):45664.

32. Moretti E, Sutera G, Collodel G. The importance of transmission electron microscopy analysis of spermatozoa: diagnostic applications and basic research. Syst Biol Reprod Med. 2016;62(3):17183.

33. Gatimel N, Moreau, Parinaud J, Léandri RD. Sperm morphology: assessment, pathophysiology, clinical relevance, and state of the art in 2017. Andrology. 2017;5(5):845-86.

34. Behrouzi B, Kenigsberg S, Alladin N, Swanson S, Zicherman J, Hong $\mathrm{SH}$, et al. Evaluation of potential protein biomarkers in 
patients with high sperm DNA damage. Syst Biol Reprod Med. 2013;59(3):153-63.

35. Mirnamniha M, Faroughi F, Tahmasbpour E, Ebrahimi P, Harchegani AB. An overview on role of some trace elements in human reproductive health, sperm function and fertilization process. Rev Environ Health. 2019;34(4):339-48.

36. Smith LB, Walker WH. The regulation of spermatogenesis by androgens. Semin Cell Dev Biol. 2014;30:2-13.

37. Thanaboonyawat I, Chantrapanichkul P, Petyim S, Kaewjunun C, Laokirkkiat P, Choavaratana R. Application of testosterone supplementation in semen to improve sperm motility in asthenozoospermic males. Arch Gynecol Obstet. 2017;296(3): 589-96.

38. Idaomar M, Guerin JF, Lorange J, Cziba JC. Stimulation of motility and energy metabolism of spermatozoa from astenozoospermic patients by 17ß-estradiol. Arch Androl. 1989;22:197-202.

39. Baldi E, Luconi M, Bonaccorsi L, Muratori M, Forti G. Intracellular events and signaling pathways involved in sperm acquisition of fertilizing capacity and acrosome reaction. Front Biosci. 2000;5: 110-23.

40. Aquila S, Sisci D, Gentile M, Carpino A, Middea E, Catalano S, et al. Towards a physiological role for cytochrome P450 aromatase in ejaculated human sperm. Hum Reprod. 2003;18(8):1650-9.

41. Cooke PS, Nanjappa MK, Ko CM, Gail S, Prins GS, Hess RA. Estrogens in male physiology. Physiol Rev. 2017;97(3):995-1043.

42. Agarwal A, Rana M, Qiu E, AlBunni H, Bui AD, Henkel R. Role of oxidative stress, infection and inflammation in male infertility. Andrologia. 2018;50(11):e13126.

43. Chao KC, Chang CC, Chiou HY, Chang JS. Serum ferritin is inversely correlated with testosterone in boys and young male adolescents: a cross-sectional study in Taiwan. PLoS One. 2015;10(12):e0144238.

44. Kernan KF, Carcillo JA. Hyperferritinemia and inflammation. Int Immunol. 2017;29(9):401-9.

45. Tremellen K, McPhee N, Pearce K, Benson S, Schedlowski M, Engler H. Endotoxin-initiated inflammation reduces testosterone production in men of reproductive age. Am J Physiol Endocrinol Metab. 2018;314(3):E206-13.

46. Ueda N, Takasawa K. Impact of inflammation on ferritin, hepcidin and the management of iron deficiency anemia in chronic kidney disease. Nutrients. 2018;10(9):1173.

47. Darbandi M, Darbandi S, Agarwal A, Sengupta P, Durairajanayagam D, Henkel R, et al. Reactive oxygen species and male reproductive hormones. Reprod Biol Endocrinol. 2018;16:87.
48. Zorn B, Ihan A, Kopitar AN, Kolbezen M, Sesek-Briski A, MedenVrtovec H. Changes in sperm apoptotic markers as related to seminal leukocytes and elastase. Reprod Biomed Online. 2010;21(1): 84-92.

49. Moretti E, Collodel G, Mazzi L, Campagna MS, Iacoponi F, Mazzi $\mathrm{L}$, et al. Resistin, interleukin-6, tumor necrosis factor-alpha, and human semen parameters in the presence of leukocytospermia, smoking habit, and varicocele. Fertil Steril. 2014;102(2):354-60.

50. Ammar O, Houas Z, Mehdi M. The association between iron, calcium, and oxidative stress in seminal plasma and sperm quality. Environ Sci Pollut Res Int. 2019;26(14):14097-105.

51. Koeva YA. Immunoreactivity for ferritin in Leydig cells of human testis. Folia Med (Plovdiv). 2002;44(3):24-6.

52. Mikuz G. The multitasking Sertoli cell. Pathologe. 2019;40(Suppl): 318-24.

53. Lécureuil C, Staub C, Fouchécourt S, Maurel MC, Fontaine I, Martinat N, et al. Transferrin overexpression alters testicular function in aged mice. Mol Reprod Dev. 2007;74(2):197-206.

54. Longini M, Moretti E, Signorini C, Noto D, Iacoponi F, Collodel G. Relevance of seminal F2-dihomo-IsoPs, F2-IsoPs and F4-NeuroPs in idiopathic infertility and varicocele. Prostaglandins Other Lipid Mediat. 2020;149:106448.

55. Lu JC, Jing J, Yao Q, Fan K, Wang GH, Feng RX, et al. Relationship between lipids levels of serum and seminal plasma and semen parameters in 631 Chinese subfertile men. PLoS One. 2016;11(1):e0146304.

56. Lu JC, Jing J, Chen L, Ge YF, Feng RX, Liang YJ, et al. Analysis of human sperm DNA fragmentation index (DFI) related factors: a report of 1010 subfertile men in China. Reprod Biol Endocrinol. 2018;16(1):23.

57. Saez Lancellotti TE, Boarelli PV, Monclus MA, Cabrillana ME, Clementi MA, Espínola LS, et al. Hypercholesterolemia impaired sperm functionality in rabbits. PLoS One. 2010;5(1):e13457.

58. Tournaye H, Krausz C, Oates RD. Novel concepts in the aetiology of male reproductive impairment. Lancet Diabetes Endocrinol. 2016;8587:1-10.

59. Bracke A, Peeters K, Punjabi U, Hoogewijs D, Dewilde S. A search for molecular mechanisms underlying male idiopathic infertility. Reprod Biomed Online. 2018;36(3):327-39.

Publisher's Note Springer Nature remains neutral with regard to jurisdictional claims in published maps and institutional affiliations. 